\title{
De las redes teóricas a las constelaciones de elementos teóricos: las prácticas científicas en la Ecología de Poblaciones"
}

\author{
From Theory-Nets to Constellations of Theory-Elements: \\ Scientific Practices in Population Ecology
}

\author{
Andoni Ibarra / Jon Larrañaga ${ }^{\dagger \ddagger}$
}

\begin{abstract}
Resumen
La metateoría estructuralista concibe las teorías científicas como redes formadas por elementos teóricos que poseen la misma estructura conceptual y están interconectados por relaciones de especialización. Además, postula que gran parte de la práctica científica tiene como fin concretar el elemento básico de estas redes añadiéndoles elementos más especializados. Así, pues, concibe el núcleo básico de elementos de una teoría como el paradigma que guía su evolución y la práctica científica normal como la adición, a redes preexistentes, de nuevos elementos teóricos. En el artículo se propone que, en la ecología de poblaciones, el patrón de la actividad científica es diferente, pues es habitual el uso conjunto de elementos de distintas redes. Se introduce la noción de "constelación teórica" para dar cuenta de estas prácticas científicas de integración, en una misma unidad epistémica, de elementos teóricos de redes distintas.

Palabras clave: redes teóricas - constelaciones teóricas - metateoría estructuralista - ecología de poblaciones - prácticas científicas
\end{abstract}

\begin{abstract}
The structuralist metatheory conceives scientific theories as networks consisting of theoryelements that have the same conceptual structures and are connected by specialization relations. Moreover, the structuralist Ansatz postulates that a considerable part of scientific practice aims at elaborating the basic elements of such nets by adding more specialized theoryelements. Thereby structuralism considers the core elements of a theory as a paradigm that guides its further evolution, and the normal practice of a theory is conceived as the addition of new theory-elements to already existing nets. The basic thesis of this paper contends that the pattern of scientific activity in population ecology is different, since it is common to use
\end{abstract}

* Recibido: 14 Enero 2011. Aceptado en versión revisada: 21 Marzo 2011.

† Departamento de Lógica y Filosofía de la Ciencia, Universidad del País Vasco/Euskal Herriko Unibertsitatea (UPV/EHU). Para contactar a los autores, por favor escriba a: andoni.ibarra@ehu.es, jlarranagapoza@ irakasle.net.

* Agradecemos a dos revisores anónimos de Metatheoria, así como a los participantes en el VII Encuentro Iberoamericano de Metateoría Estructuralista, y especialmente a Diego Méndez, sus comentarios críticos y sugerencias a versiones previas de esta contribución. El trabajo se ha desarrollado parcialmente en el marco del proyecto de investigación "Modelos y representación en ciencias formales y fácticas. Análisis históricos y conceptuales" (PICTR2006, № 2007), financiado por la Agencia Nacional de Promoción Cientifica y Tecnológica de la República Argentina.

Metatheoria 1(2)(2011): 167-193. ISSN 1853-2322.

(C) Editorial de la Universidad Nacional de Tres de Febrero. Publicado en la República Argentina. 
theory-elements from distinct nets. In order to take into account these practices the concept of "theoretical constellation" is introduced intending to capture the idea of an epistemic unification of theory-elements that come from different nets.

Keywords: theory-nets - theoretical constellations - structuralist metatheory - population ecology - scientific practices

\section{Introducción}

La ecología de poblaciones estudia el modo en que diversos factores (la competencia, la depredación, las migraciones, la distribución espacial y temporal de los recursos, etc.) hacen que el tamaño de las poblaciones cambie. Este estudio se ha visto fuertemente condicionado por el hecho de que las poblaciones son sistemas muy complejos en cuyas dinámicas influyen muchos y muy diversos factores y se ha basado, fundamentalmente, en la construcción, análisis y puesta en común de elementos teóricos de muy diferentes características. Ante la complejidad de los sistemas ecológicos y para evitar los problemas asociados a la excesiva complejidad de los elementos teóricos, los ecólogos han abandonado la pretensión de construir elementos teóricos que incluyan a estos factores de manera exhaustiva. En su lugar, han optado por una aproximación pluralista, en la que se utilizan elementos teóricos o representaciones de muy diferentes tipos, que incluyen la influencia de una cantidad limitada de factores o fenómenos, y cuyos comportamientos son comparados entre sí para obtener conocimiento sobre cómo esos factores o fenómenos influyen en las dinámicas de las poblaciones. Dicho de otro modo, el procedimiento por el cual los ecólogos teóricos han tratado de comprender la influencia de diversos factores en las dinámicas de las poblaciones ha sido el de la construcción de elementos teóricos diferentes, que incluyen o no determinados factores o procesos o que los incluyen de maneras diferentes, y compararlos entre sí. Esta práctica, característica de la ecología de poblaciones, replantea algunos aspectos bien fijados de la metateoría estructuralista.

Según esta metateoría, las teorías científicas suelen ser, al menos la mayoría de ellas, redes formadas por cierta cantidad de elementos, que poseen la misma estructura básica y se encuentran interconectados por medio de relaciones de especialización (Balzer, Moulines \& Sneed 1987, pp. 167-204, Moulines 1996, 2010). En estas redes teóricas se identifican uno o muy pocos elementos básicos, definidos por leyes fundamentales de carácter muy esquemático, y una cantidad más o menos grande de elementos teóricos más especializados, que se construyen mediante un proceso de especificación sucesiva de los elementos teóricos básicos. Estos elementos teóricos secundarios se definen o caracterizan por otras leyes y por restricciones que se añaden a las leyes y restricciones básicas. Este proceso da lugar a una serie de elementos teóricos cada vez más especializados, cuyo dominio de aplicaciones pretendidas es, también, cada vez más limitado. Estas redes son, además, genidénticas, en el sentido de que, mientras que el elemento básico de la teoría o núcleo duro de aplicaciones paradigmáticas se preserva a lo largo de toda la vida de la teoría, muchas de las especializaciones derivadas de este 
elemento básico o núcleo duro aparecen y desaparecen dependiendo de diversos factores o circunstancias. ${ }^{1}$

Desde un punto de vista formal, podemos definir una red teórica idealizada (idealized theory-net) como sigue (Balzer, Moulines \& Sneed 1987, pp. 172-177):

$\mathrm{N}$ es una red teórica idealizada syss existen $\overline{\mathbf{T}}$ y $\sigma$ tales que

(1) $\mathrm{N}=\langle\overline{\mathbf{T}}, \sigma\rangle$

(2) $\overline{\mathbf{T}}$ es un conjunto finito, no-vacío, de elementos teóricos

(3) $\sigma \subseteq \overline{\mathbf{T}} \times \overline{\mathbf{T}}$ es la relación de especialización (restringida a $\overline{\mathbf{T}}$ ).

Sin embargo, esta definición de red teórica es excesivamente general, ya que permite la existencia de redes teóricas que carecen de estructura jerárquica, cuando, de acuerdo con la metateoría estructuralista, las redes realmente existentes en la ciencia tienen estructura jerárquica. Esta condición introduce una restricción en la definición anterior. Llamamos red teórica conectada (connected theory-net) a la red definida de la siguiente manera:

$\mathbf{N}$ es una red teórica conectada syss existen $\overline{\mathbf{T}}$ y $\sigma$ tales que

(1) $\mathbf{N}=\langle\overline{\mathbf{T}}, \sigma\rangle$ es una red teórica idealizada

(2) para toda $\mathbf{T}_{\mathbf{i}}, \mathbf{T}_{\mathbf{j}} \in \overline{\mathbf{T}}$ existen $\mathbf{T}_{\mathrm{k}_{1}}, \ldots, \mathbf{T}_{\mathrm{k}_{\mathrm{n}}}$ tales que:

$$
\left(\mathbf{T}_{\mathrm{i}} \sigma \mathbf{T}_{\mathrm{k}_{1}} \vee \mathbf{T}_{\mathrm{k}_{1}} \sigma \mathbf{T}_{\mathrm{i}}\right) \wedge \ldots \wedge\left(\mathbf{T}_{\mathrm{j}} \sigma \mathbf{T}_{\mathrm{k}_{\mathrm{n}}} \vee \mathbf{T}_{\mathrm{k}_{\mathrm{n}}} \sigma \mathbf{T}_{\mathrm{j}}\right)
$$

Esta segunda definición exige que dos elementos teóricos cualesquiera de $\mathrm{N}$ sean especializaciones de otro elemento de orden superior o tengan una especialización común. Obviamente, en toda red teórica conectada habrá elementos teóricos que no sean especializaciones de ningún otro elemento teórico (normalmente uno por cada red). Estos serían los elementos teóricos básicos de la red, los elementos de los que, por medio de relaciones de especialización, se derivan todos los demás elementos de la red teórica. De esta definición se sigue, además, que todos los elementos de una red teórica conectada poseen los mismos modelos potenciales $\left(\mathbf{M}_{\mathbf{p}}\right)$ y modelos potenciales parciales $\left(\mathbf{M}_{\mathbf{p p}}\right)$.

La metateoría estructuralista postula, también, que gran parte de la actividad de los científicos tiene como fin concretar, progresivamente y en diversas direcciones, el núcleo inicial de estas teorías, añadiendo a las redes teóricas elementos cada vez mázs especializados. Esto último sugiere que la metateoría estructuralista concibe el núcleo básico de elementos teóricos que conforman una teoría como el paradigma (sensu Kuhn) que guía los subsiguientes desarrollos teóricos. Así pues, durante los periodos de ciencia normal el desarrollo teórico consistiría, fundamentalmente, en añadir en la red teórica nuevos elementos cada vez más especializados (evolución teórica).

Ciertamente, la metateoría estructuralista contempla el hecho de que las teorías no son entidades aisladas y reconoce la existencia de vínculos interteóricos que relacionan entre sí elementos teóricos de distintas redes y dan lugar

\footnotetext{
${ }^{1}$ Esta imagen de las redes ha sido corroborada en un gran número de teorías reconstruidas como redes; ver Diederich, Ibarra \& Mormann $(1989,1994)$.
} 
a estructuras formadas por elementos de varias redes teóricas: los denominados holones teóricos (Balzer, Moulines \& Sneed 1987, pp. 386-423). Sin embargo, al poner el énfasis en la reconstrucción de teorías, en la metateoría estructuralista prima la visión de la práctica científica en periodos de ciencia normal como consistente en la ampliación de las redes preexistentes mediante la adición de nuevos elementos más especializados a los elementos teóricos. Ello elucidaría la intuición kuhniana de la actividad "normal" del científico "en el marco de un paradigma”.

En la ecología de poblaciones, el patrón de las prácticas científicas parece ser algo diferente al bosquejado hasta aquí. También en otras disciplinas, pero singularmente en esta, la práctica teórica fundamental consiste en la construcción y el análisis comparativo de elementos teóricos. Sin embargo, a menudo los elementos teóricos que se construyen y se comparan entre sí no pueden ser considerados desarrollos o especializaciones de una única red teórica. Por el contrario, en las prácticas normales de la ecología de poblaciones se utilizan conjuntamente elementos teóricos que no pueden ser considerados especializaciones de una única red teórica preexistente. Estos elementos teóricos no suelen ser objetos aislados, sino que son producidos y analizados en un marco en el que es fundamental la comparación o puesta en común con otros elementos de otras redes teóricas. Todo esto sugiere que en la ecología de poblaciones las prácticas "normales" integran elementos pertenecientes a diferentes redes teóricas. Estos elementos teóricos relacionados estrechamente en las prácticas constituyen asociaciones que aquí denominaremos constelaciones teóricas. Las constelaciones teóricas se utilizan para el análisis de un determinado dominio de aplicación, por ejemplo, el estudio de la dinámica de la población $X$.

Para hacer razonable esta interpretación procederemos como sigue: en la sección siguiente mostraremos un esbozo de la red teórica asociada a la denominada teoría logística clásica; luego, en la sección 3, expondremos cómo la consideración discreta del tiempo permite generar una red de elementos alternativa a la anterior y cómo el conocimiento robusto relativo a las poblaciones naturales se obtiene a partir de prácticas de comparación de elementos teóricos de las dos redes, esto es, considerando las unidades que denominamos constelaciones teóricas. Finalmente, en la sección 4, se analizará una red independiente de estas dos, pues consta de elementos teóricos que, a diferencia de la teoría logística clásica y su alternativa en tiempo discreto, tiene en cuenta cada uno de los individuos que conforman la población y que, sin embargo, es utilizada para abordar las mismas cuestiones poblacionales que analizan las dos teorías clásicas ya mencionadas. A partir de esa reconstrucción se mostrará cómo las prácticas científicas integran elementos de esta red más compleja, junto con elementos más sencillos de otras redes, en constelaciones que procuran la obtención de resultados robustos. Concluiremos mostrando la conveniencia de incorporar a la teoría de la ciencia la unidad epistémica de las constelaciones teóricas para dar cuenta de la naturaleza de las prácticas científicas en algunos dominios, como el de la ecología de poblaciones. 


\section{La teoría logística clásica y su estructura}

La teoría logística para el estudio de los factores que regulan el crecimiento de una población es un sencillo ejemplo del tipo de teorías que puede hallarse en la ecología de poblaciones. En el origen de esta teoría se encuentra la conocida ecuación logística de Verhulst (Kingsland 1995, p. 65):

$$
\frac{d N}{d t}=r N\left(1-\frac{N}{K}\right)
$$

donde $N$ es el tamaño de la población, $t$ el tiempo, $r$ la tasa de natalidad per capita y $K$ un parámetro que limita el tamaño máximo que puede alcanzar la población y que se conoce como capacidad de carga de la población. Así pues, en su forma de ecuación diferencial, este elemento teórico representa la velocidad de crecimiento de la población como función del tamaño o de la densidad de la población.

\section{Figura 1}

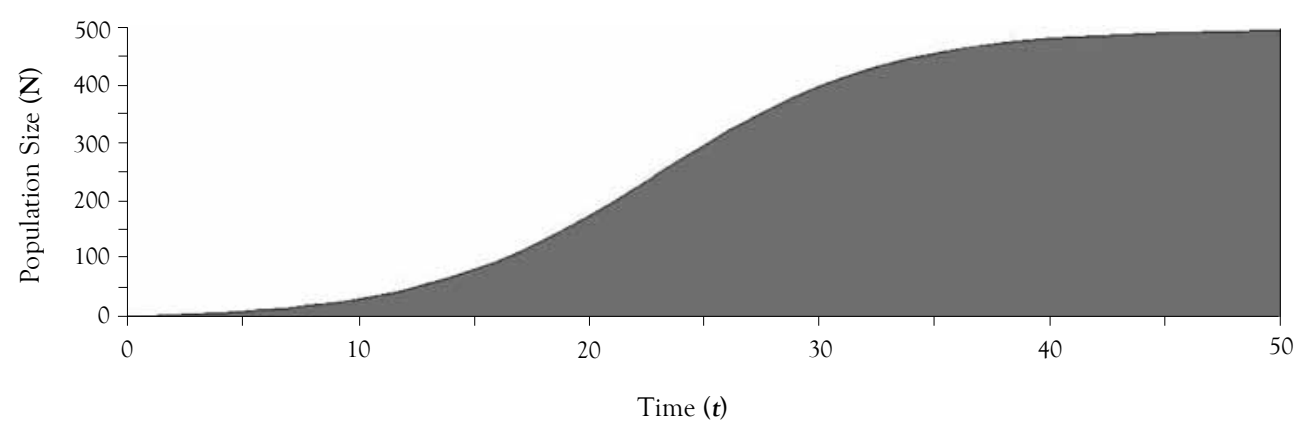

La versión integral de la ecuación logística de Verhulst representa el tamaño de la población frente al tiempo dando lugar a la conocida curva sigmoidea donde se observa que: (1) cuando la densidad es baja la población crece lentamente; (2) la tasa de crecimiento de la población aumenta con la densidad hasta que esta llega a $K / 2$, densidad a partir de la cual la velocidad de crecimiento de la población va disminuyendo gradualmente; y (3) el tamaño de la población se acerca asintóticamente a $K$.

Una de las críticas más habituales que se hace al elemento teórico de Verhulst es la de su escaso realismo, pues su comportamiento en nada se parece a lo que se observa en las poblaciones naturales (Hall 1988). Habitualmente, se explica la discrepancia entre lo predicho y lo observado alegando que el modelo logístico es muy poco realista, muy idealizado o muy abstracto y se recuerda que el elemento teórico asociado a él representa las poblaciones como entes cerrados en los que no se producen movimientos migratorios, todos los individuos de la población son iguales, la capacidad de carga es constante, la relación entre los cambios de densidad y los cambios en la tasa de crecimiento de la población es lineal y, además, estos cambios se producen de manera inmediata (Gotelli 2001, pp. 30-31). 
El hecho de que el elemento teórico de Verhulst no represente adecuadamente el modo en que la densidad influye en las dinámicas de las poblaciones está en el origen de la construcción de otros elementos teóricos en los que se integran algunas variaciones relativas a diversos parámetros (generando así líneas de especialización en la red teórica, en el sentido estipulado por la concepción estructuralista). Un ejemplo es el elemento teórico theta-logístico, que explora la posibilidad de que la relación entre la densidad y la tasa de crecimiento no sea lineal y cuya ley tiene la siguiente forma:

$$
\frac{d N}{d t}=r N\left[1-\left(\frac{N}{K}\right)^{\theta}\right]
$$

Como se puede ver intuitivamente en sus leyes respectivas, la única diferencia formal entre este nuevo elemento teórico y el de Verhulst es la inclusión de un nuevo parámetro, $\theta, y$, obviamente, el comportamiento de este segundo modelo depende en gran medida del valor de ese nuevo parámetro. Es decir, es atribuyendo diferentes valores al parámetro $\theta$ como se exploran las consecuencias de que la relación entre los cambios en densidad y los cambios en la tasa de crecimiento de la población no sea lineal. Obsérvese que el elemento teórico de Verhulst puede considerarse una especialización del elemento theta-logístico para $\theta=$ $1 \mathrm{o}$, dicho de otro modo, que el elemento theta-logístico es una generalización de aquel (Turchin 2003, pp. 49-50).

Otros elementos se construyen a partir de una línea de especialización que tiene en cuenta el denominado efecto Allee. Existen indicios de que en las poblaciones muy pequeñas la tasa reproductiva de los individuos suele ser menor que la observada en poblaciones mayores, debido a que las interacciones cooperativas entre individuos (en la búsqueda de alimentos, la defensa frente a los depredadores, etc.) suelen fallar. Este fenómeno es conocido como efecto Allee y, cuando este es muy marcado, puede ocurrir que, por debajo de un umbral de densidad mínimo, la población comience a decrecer y se extinga (Lande, Engen \& Saether 2003, p. 36). En esta línea de especialización dependiente del efecto Allee el elemento teórico más sencillo es el asociado al conocido como "modelo logístico con limitación de densidad mínima", cuya ley es:

$$
\frac{d N}{d t}=r N\left(1-\frac{N}{K}\right)\left(1-\frac{C}{N}\right)
$$

Este elemento, a diferencia del elemento teórico de Verhulst, presenta un componente (1-C/N), donde $C$ es un umbral de densidad crítica por debajo del cual el tamaño de la población comienza a decrecer. A causa de este nuevo término, el elemento teórico describe un punto de equilibrio inestable en $N=C$, ya que cualquier incremento de $N$ por encima de $C$ da lugar a un proceso de crecimiento que acerca la densidad de la población a $K$, pero cualquier fluctuación que haga descender la densidad por debajo de $C$ lleva a la extinción de la población (Wimsatt 1980).

Como puede deducirse de los ejemplos mostrados, la red logística clásica está compuesta por elementos teóricos con diferentes líneas de especialización en las que las variables principales son la densidad de población, $N$, y el tiempo, $t$, y 
siempre aparecen como parámetros la capacidad de carga $K$, más un parámetro adicional que representa la capacidad reproductora de los componentes de la población, habitualmente, la tasa de crecimiento per capita, $r$. No es nuestro objetivo desarrollar una reconstrucción formal precisa de la red teórica logística, pero sí queremos mostrar un esbozo de reconstrucción que sirva como sostén provisional para los argumentos que seguidamente se desarrollarán.

La estructura del marco conceptual de la teoría logística, identificada con la clase de los modelos potenciales del elemento logístico de Verhulst, se define como sigue:

$\mathbf{M}_{\mathbf{p}}(\mathbf{L O G V}): x$ es una población logística de Verhulst potencial $\left(x \in \mathbf{M}_{\mathbf{p}}(\mathbf{L O G V})\right)$ syss existen $N, T, r, k$ y $f$ tales que

(1) $x=\langle N, T, r, k, f\rangle$

(2) $N$ es un conjunto finito, no vacío y $N=\{n \in \mathbb{R}: 0 \leq n \leq k\}$

(3) $T$ es un conjunto y $T \subseteq \mathbb{R}$

(4) $k \in \mathbb{N}$

(5) $r \in \mathbb{R}$

(6) $f: T \rightarrow N$

donde $N$ es el tamaño poblacional y $T$ el tiempo. $r$ y $k$ son, respectivamente los parámetros conocidos como tasa de crecimiento per capita y capacidad de carga o límite máximo que la población no podrá superar. ${ }^{2}$ La función $f$ asigna un tamaño poblacional $(n)$ a cada instante de tiempo $(t)$; tiene que ser una función continua y derivable, ya que la velocidad de crecimiento se define como la derivada del tamaño poblacional con respecto al tiempo (esto exige, aunque resulte contraintuitivo, atribuir valores reales a los elementos del conjunto $N$, esto es, a los tamaños de la población).

La clase de los modelos actuales del mencionado elemento teórico se define de la siguiente manera:

M(LOGV): $x$ es una población logística de Verhulst $(x \in \mathbf{M}(\mathbf{L O G V}))$ syss existen $N$, $T, r, k$ y $f$ tales que

(1) $x=\langle N, T, r, k, f\rangle$

(2) $x \in \mathbf{M}_{\mathbf{p}}(\mathbf{L O G V})$

(3) $D f=r n(1-n / k)$

donde $D f$ denota la derivada de la función $f$.

Por su parte, el elemento teórico theta-logístico (THLOG) presenta una estructura prácticamente idéntica, salvo por el hecho de que incluye un parámetro más, el parámetro $\theta$, que, como se ha dicho ya, determina el tipo de relación existente entre los cambios en el tamaño de la población y los cambios en su velocidad de crecimiento y que vendría dado por un número real. Así, la clase de los modelos potenciales de este elemento teórico tiene la siguiente estructura:

\footnotetext{
${ }^{2}$ Dado que la densidad de una población es una cantidad de individuos, los ecólogos solo atribuyen valores enteros y positivos a este parámetro; cualquier otro valor resulta contraintuitivo.
} 
$\mathbf{M}_{\mathbf{p}}$ (THLOG): $x$ es una población theta-logística potencial ( $x \in \mathbf{M}_{\mathbf{p}}$ (THLOG)) syss existen $N, T, r, k, \theta$ y $f$ tales que

(1) $x=\langle N, T, r, k, \theta, f\rangle$

(2) $N$ es un conjunto finito, no vacío y $N=\{n \in \mathbb{R}: 0 \leq n \leq k\}$

(3) $T$ es un conjunto y $T \subseteq \mathbb{R}$

(4) $k \in \mathbb{N}$

(5) $r \in \mathbb{R}$

(6) $\theta \in \mathbb{R}$

(7) $f: T \rightarrow N$

Una población theta-logística es toda población que satisface las siguientes condiciones:

M(THLOG): $x$ es una población theta-logística $(x \in \mathbf{M}($ THLOG $))$ syss existen $N$, $T, r, k, \theta$ y $f$ tales que

(1) $x=\langle N, T, r, k, \theta, f\rangle$

(2) $x \in \mathbf{M}_{\mathbf{p}}$ (THLOG)

(3) $D f=r n\left[1-\left(\frac{n}{k}\right)^{\theta}\right]$

En cambio, para ser una población logística equipada con el efecto Allee (LOGA), una población debe incluir, además de los componentes de una población logística de Verhulst, un parámetro adicional $c$, mayor que 0 y menor que $k$, que determina un umbral de densidad crítica por debajo del cual el crecimiento de la población sería negativo y por encima del cual el crecimiento sería positivo. Así pues, una población logística Allee (LOGA) potencial se define como sigue: $\mathbf{M}_{\mathbf{p}}$ (LOGA): $x$ es una población logistica Allee potencial $\left(x \in \mathbf{M}_{\mathbf{p}}(\mathbf{L O G A})\right)$ syss existen $N, T, r, k$, c y $f$ tales que

(1) $x=\langle N, T, r, k, c, f\rangle$

(2) $N$ es un conjunto finito, no vacío y $N=\{n \in \mathbb{R}: 0 \leq n \leq k\}$

(3) $T$ es un conjunto y $T \subseteq \mathbb{R}$

(4) $k \in \mathbb{N}$

(5) $r \in \mathbb{R}$

(6) $c \in \mathbb{N}$ y $0<c<k$

(7) $f: T \rightarrow N$

Los modelos actuales de este elemento teórico satisfacen las siguientes condiciones:

M(LOGA): $x$ es una población logística Allee $(\mathrm{x} \in \mathbf{M}($ LOGA $)$ ) syss existen $N, T, r$, $k, c$ y $f$ tales que
(1) $x=\langle N, T, r, k, c, f\rangle$
(2) $x \in \mathbf{M}_{\mathbf{p}}$ (LOGA)
(3) $D f=r n(1-n / k)(1-c / n)$ 
A la vista de estas reconstrucciones tentativas, parece claro que, en la medida en que los tres elementos teóricos mencionados comparten en sus núcleos básicos, $\mathbf{M}_{\mathrm{p}}$, los componentes $N, T, k$ y $r$, así como los conjuntos $\mathbb{N}$ y $\mathbb{R}$, pueden ser considerados integrantes de una misma red teórica a la que denominaremos red logística. El fragmento de la red logística que integra estos tres elementos tiene la siguiente forma:

Figura 2

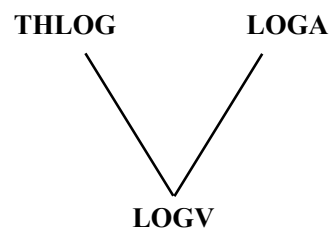

Fragmento de red teórica en el que se integran el elemento teórico theta logístico el elemento teórico logístico con efecto Allee y el elemento logístico de Verhulst.

El elemento logístico de Verhulst puede ser considerado un caso particular tanto del elemento teórico theta-logístico (concretamente para $\theta=1$ ), como del elemento que incluye el efecto Allee (en este caso, para $\mathrm{c}=0$ ).

Es más, podríamos integrar en la red teórica logística otros muchos elementos teóricos de mayor o menor complejidad, siempre y cuando contengan entre sus variables la densidad poblacional, $N$, y el tiempo, $t$, y entre sus parámetros la capacidad de carga, $K$. Así, por ejemplo, puede integrarse el siguiente elemento, que corresponde a la ley propuesta por Gause en 1934 (Jensen 1975):

$$
\frac{d N}{d t}=b N\left(1-\frac{N}{K}\right)
$$

donde la tasa de crecimiento per capita, $r$, es sustituida por la tasa de natalidad per capita, $b .^{3}$ Este último elemento teórico puede integrarse en la red logística de la siguiente manera:

Figura 3

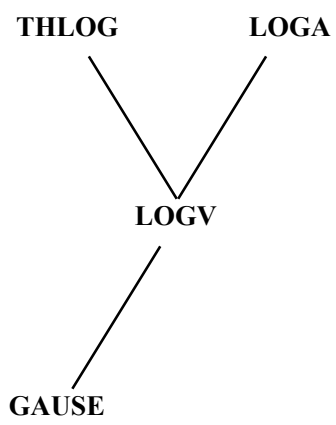

Posición que ocupa el elemento teórico de Gause en la red teórica logística.

\footnotetext{
${ }^{3}$ Téngase en cuenta que la tasa de crecimiento per capita, $r$, puede descomponerse en dos componentes, la tasa de na-
} talidad per capita, $b$, y la tasa de mortalidad per capita, $d$, de modo que $r=b-d$ (Vandermeer $\&$ Goldberg 2003, p. 6). 
Es decir, el elemento teórico de Gause puede considerarse una especialización del elemento logístico de Verhulst aplicable en aquellos casos en los que el efecto de la tasa de mortalidad sobre la población fuera despreciable.

De modo semejante, pueden incluirse en la red logística muchos otros elementos, en los que las variables principales sean la densidad de población y el tiempo y que, junto a un parámetro que dé cuenta del modo como los componentes de la población aumentan en cantidad, incluya otro que marque un límite de tamaño o densidad máximo que la población no puede superar.

Sin embargo, las prácticas científicas de los ecólogos no se limitan a incorporar nuevos elementos a la red logística. Característicamente, al investigar la influencia de diferentes factores en las dinámicas de las poblaciones naturales, los ecólogos recurren, de manera sistemática, a elementos de diferentes redes teóricas, que utilizan de manera conjunta, bien para mostrar el contraste entre las conclusiones a que dan lugar, bien para mostrar la coincidencia de resultados. En la ecología de poblaciones teórica, una de las estrategias más habituales para comprender la influencia de determinados factores en el comportamiento de las poblaciones es la comparación o puesta en común de elementos teóricos integrados en redes teóricas diferentes. Es decir, en sus prácticas normales los ecólogos hacen uso simultáneo de elementos teóricos de diferentes redes, combinándolos entre sí. Esta combinación permite la descripción y explicación de los hechos bajo escrutinio.

\section{La comparación entre elementos teóricos como práctica genuina en la ecología de poblaciones. El papel de las constelaciones teóricas}

Como ha quedado indicado, la práctica característica en la ecología de poblaciones es la de combinar diversos elementos teóricos para tratar de dar cuenta del papel de diferentes factores o procesos en la dinámica de poblaciones. Por eso, pese a tener escasa capacidad de predicción o mostrar comportamientos que no se ajustan bien a lo observado en las poblaciones naturales, los elementos teóricos son considerados útiles para esa labor. Se considera que estos elementos, pese a ser representaciones demasiado burdas de las poblaciones naturales, pueden servir, sobre todo si se utilizan en combinación con otros elementos, para extraer conclusiones robustas sobre la influencia de diferentes factores en las dinámicas de las poblaciones (Levins 1966, Odenbaugh 2003, Weisberg 2006). A continuación mostraremos cómo esas combinaciones involucran elementos de redes teóricas diferentes.

Una estrategia de combinación posible entre estos elementos teóricos interreticulares se genera a partir de la consideración del tiempo transcurrido entre un cambio en la densidad poblacional y la manifestación de sus efectos. El elemento logístico de Verhulst representa las poblaciones naturales como agregados en los que la incorporación o la desaparición de cierta cantidad de individuos tienen 
como consecuencia un cambio en la tasa de crecimiento de la población. Además, de acuerdo con este elemento teórico, dicho cambio se produce de manera inmediata, por lo que se considera que el elemento logístico de Verhulst no incluye retrasos temporales. Sin embargo, parece más razonable suponer que, con frecuencia, las consecuencias de los cambios de tamaño de la población no son inmediatas, sino que se manifiestan transcurrido un período. Los ecólogos han construido elementos teóricos que, de una manera u otra, incluyen la influencia de estos retrasos temporales y, por tanto, sirven para estudiar sus efectos.

Uno de los más sencillos es el denominado modelo de Hutchinson, cuya ley es:

$$
\frac{d N}{d t}=r N\left(1-\frac{N_{t-\tau}}{K}\right)
$$

La estructura de los modelos potenciales del elemento teórico asociado a esta ley viene definida de la siguiente manera:

$\mathbf{M}_{\mathbf{p}}(\mathbf{L O G H}): x$ es una población logística de Hutchinson (o una población con retraso temporal) potencial $\left(x \in \mathbf{M}_{\mathbf{p}}(\mathbf{L O G H})\right)$ syss existen $N, T, r, k, \tau$ y $f$ tales que

(1) $x=\langle N, T, r, k, \tau, f\rangle$

(2) $N$ es un conjunto finito, no vacío y $N=\{n \in \mathbb{R}: 0 \leq n \leq k\}$

(3) $T$ es un conjunto y $T \subseteq \mathbb{R}$

(4) $k \in \mathbb{N}$

(5) $r \in \mathbb{R}$

(6) $\tau \in \mathbb{R}^{+}$

(7) $f: T \rightarrow N$

donde $\tau$ es el retraso temporal.

Los modelos actuales de este elemento teórico satisfacen las siguientes condiciones:

M(LOGH): $x$ es una población logística $(x \in \mathbf{M}(\mathbf{L O G H}))$ syss existen $N, T, r, k, \tau$ y $f$ tales que

(1) $x=\langle N, T, r, k, \tau, f\rangle$

(2) $x \in \mathbf{M}_{\mathbf{p}}(\mathbf{L O G H})$

(3) $D f=r n\left(1-n_{t-\tau} / k\right)$

Obsérvese que la ley de este último elemento teórico es prácticamente idéntica a la del elemento teórico de Verhulst, salvo que, para calcular la tasa de crecimiento de la población en un instante $t$, tiene en cuenta, además de la densidad de la población en ese instante ( $n$ en la reconstrucción modeloteórica), la densidad de la población en un instante anterior $t-\tau\left(n_{t-\tau}\right.$ en la reconstrucción modeloteórica), donde $\tau$ es el tiempo que transcurre desde que ocurre un cambio en la densidad de la población hasta que se manifiestan sus efectos.

Esta similitud de estructura indica que el elemento teórico de Hutchinson también forma parte de la red logística y el hecho de que el elemento logístico de Verhulst pueda ser considerado un caso límite del elemento teórico de Hutchinson para el caso en el que el retraso temporal sea inexistente $(\tau=0)$ sugiere que 
la configuración del fragmento de red logística en el que está incluido este último elemento es el siguiente:

Figura 4

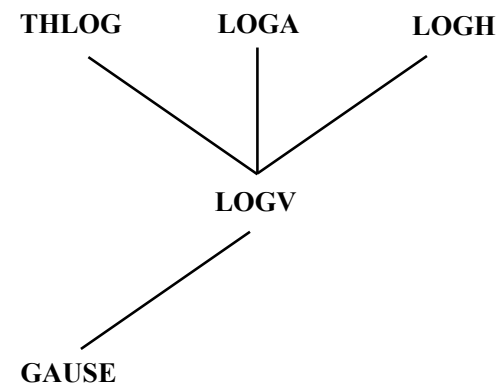

Posición que ocupa el elemento teórico de Hutchinson en la red logística.

Es más, este fragmento de red logística sugiere que existe al menos un elemento teórico primitivo del que se especializarían el resto de los elementos teóricos hasta ahora mencionados (al lado de las conexiones entre los elementos teóricos se indican las transformaciones que dan lugar al elemento teórico del nivel inferior). ${ }^{4}$

Figura 5

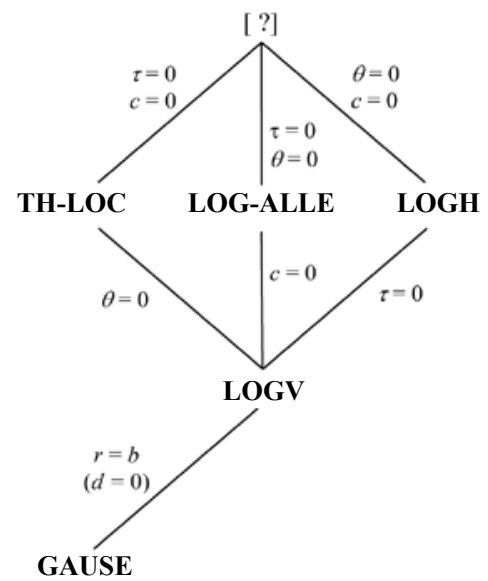

Fragmento de red teórica en el que se integran todos los elementos teóricos en tiempo continuo analizados y se muestra la posición que ocuparía el elemento teórico del que todos ellos serían especializaciones.

\footnotetext{
${ }^{4}$ La figura representa, como acaba de indicarse, un fragmento de la red. De ahí que la imagen resultante no se corresponda con la habitual del árbol reticular. No es nuestro objetivo reconstruir enteramente la red logística sino mostrar la naturaleza reticular de las relaciones entre los elementos logísticos y cómo ellos son utilizados en las prácticas de los ecólogos.
} 
Ahora bien, desde el punto de vista ecológico, lo interesante de este elemento teórico es que sugiere que la existencia de retrasos temporales tiene un efecto desestabilizador en la población (Gotelli 2001, pp. 33-34; Figura 6).
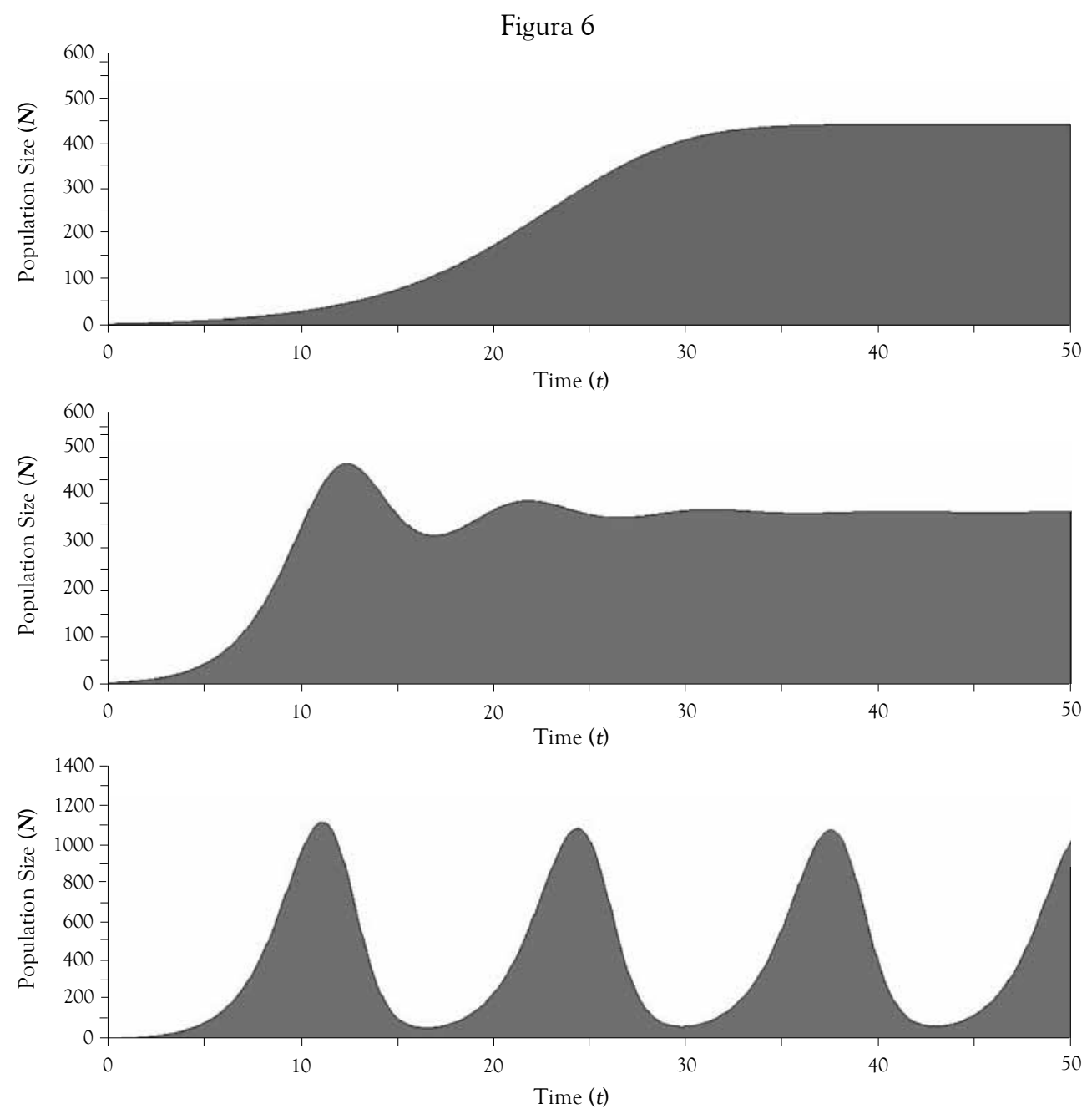

Comportamiento del elemento teórico logístico con retraso temporal para diferentes valores de $r$ y $\tau$. El retraso temporal tiene un efecto desestabilizador, en el sentido de que cuando el valor de $\tau$ supera un determinado umbral la dinámica de la población pasa de ser sigmoidea a estar caracterizada por ciclos amortiguados o por ciclos con límites estables. De arriba abajo, primer gráfico $r=0,2$ y $\tau=2$; segundo $r=0,5$ y $\tau=2$ y tercero $r=0,6$ y $\tau=3$.

El elemento teórico de Hutchison es el más sencillo de los que pueden construirse considerando la introducción de retrasos temporales en una ley diferencial. Ahora bien, la investigación de los factores que afectan a las dinámicas de las poblaciones ha dado lugar a otros elementos teóricos que no forman parte de la red logística y que, sin embargo, han sido utilizados junto con elementos de esa 
red para analizar la influencia de los retrasos temporales en las dinámicas de las poblaciones naturales. Dicho de otro modo, el estudio de los efectos desestabilizadores de los retrasos temporales se ha llevado a cabo comparando entre sí elementos de diferentes redes teóricas, lo que ha permitido afianzar la conclusión de que los retrasos temporales tienen una influencia desestabilizadora en las poblaciones. En otras palabras, es la comparación de elementos teóricos pertenecientes a diferentes redes lo que permite afirmar la robustez de la conclusión de que los retrasos temporales tienen efectos desestabilizadores en las poblaciones.

En efecto, las ecuaciones diferenciales características de los elementos de la red logística no son la única manera de representar la dinámica de las poblaciones. Existen otras posibilidades, una de las cuales, tan habitual como el uso de ecuaciones diferenciales, es el uso de ecuaciones en diferencias. En este segundo tipo de ecuaciones, el tratamiento del tiempo es diferente, ya que este es tratado como una variable discreta, mientras que en las ecuaciones diferenciales es tratado como variable continua. Por este motivo, en la literatura ecológica, a las ecuaciones en diferencias se las denomina, también, "modelos en tiempo discreto", mientras que los elementos teóricos asociados a leyes dadas por ecuaciones diferenciales son conocidos como "modelos en tiempo continuo".

Pues bien, podemos reconstruir un buen número de elementos teóricos asociados a leyes dadas por ecuaciones en diferencias en el marco de una nueva red, distinta de la logística. De hecho, es posible construir análogos en tiempo discreto de prácticamente todos los elementos teóricos de la red logística, aunque, en general, se suelen construir a partir de los elementos teóricos más generales, derivándolos al modo de relaciones de especialización. Actualmente, los elementos teóricos en tiempo discreto son una de las principales herramientas de la ecología de poblaciones, ampliamente utilizados, tanto con fines teóricos como en la gestión ambiental. Además, por el mero hecho de estar asociados a ecuaciones en diferencias, estos elementos teóricos en tiempo discreto incorporan ya la influencia del retraso temporal, aunque suelen existir variaciones notables en la forma en que incluyen dicho retraso. Seguidamente, mostraremos algunos de estos elementos. ${ }^{5}$

\footnotetext{
${ }^{5}$ La red de elementos que presentamos es controvertible. Podría argüirse, como lo hace uno de los revisores, que los elementos teóricos con el tiempo como variable discreta podrían reconstruirse como formando parte, junto con los elementos de la red logística, de una red que subsumiría a todos ellos y sería generada a partir de un elemento teórico con muy poco contenido empírico (p.e., el número de miembros de una población en un momento dado depende de su número en algún momento anterior). La dependencia de la forma matemática concreta que asume el tiempo, continua o discreta, fijaría una de las especializaciones de esa red subsumidora de todos los elementos.

Como se viene sosteniendo, el objetivo del artículo es ofrecer una imagen de las prácticas científicas más plausible que las sugeridas por la ciencia normal de Kuhn o sus secuelas estructuralistas. Los ecólogos utilizan profusamente el concepto teoría y, más aún, marco teórico de manera imprecisa. Para ellos, los marcos teóricos son conjuntos más o menos diversos de elementos teóricos con los que analizan cuestiones tales como las dinámicas de poblaciones aisladas, las interacciones entre dos poblaciones de especies diferentes, las dinámicas de las comunidades insulares, la influencia de la distribución espacial de los individuos en la persistencia de las poblaciones que estos forman, etc. El hecho característico de muchas prácticas científicas, como las ecológicas, es que habitualmente esas cuestiones se abordan apelando a conjuntos conformados por elementos teóricos del marco logístico, de sus representaciones del tiempo como variable discreta, etc. Y, frecuentemente, esos marcos son diferentes según los diversos autores que los aplican y que muestran preferencias distintas por un marco u otro.
} 
Existen al menos tres análogos en tiempo discreto del elemento logístico de Verhulst (Turchin 2003, pp. 52-55). Uno de ellos es el asociado al que entre los ecólogos es conocido como modelo de Ricker, que fue desarrollado como herramienta para la gestión de pesquerías, pero ha sido utilizado también con fines teóricos, y cuya ley tiene la forma:

$$
N_{t+1}=\lambda_{0} N_{t} e^{\left(-b N_{t}\right)}
$$

donde $N$ es el tamaño o la densidad de población y los subíndices $t$ y $t+1$ recuerdan que el tiempo es tratado como una variable discreta; el parámetro $\lambda_{0}$ es una tasa de crecimiento independiente de la densidad, y $b$ es una medida de la dependencia de la densidad $\left(b=\lambda_{\sigma} / K\right)$.

Los modelos potenciales del elemento teórico de Ricker satisfacen las siguientes condiciones:

$\mathbf{M}_{\mathbf{p}}(\mathbf{R}): x$ es una población de tipo Ricker potencial $\left(x \in \mathbf{M}_{\mathbf{p}}(\mathbf{R})\right)$ syss existen $N, T, \lambda_{0}$, $k$ y $b$ tales que

(1) $x=\left\langle N, T, \lambda_{0}, k, b\right\rangle$

(2) $N$ es un conjunto finito, no vacío y $N \subseteq \mathbb{N}$

(3) $T$ contiene exactamente dos elementos $\left(T=\left\{t_{1}, t_{2}\right\}\right)$

(4) $\lambda_{0} \in \mathbb{R}$

(5) $k \in \mathbb{N}_{\text {y }} k<|N|$

(6) $b=\lambda / k$

(7) Trunc: $\mathbb{R} \rightarrow \mathbb{N}$

donde Trunc: $\mathbb{R} \rightarrow \mathbb{N}$ es una función que redondea un número real a su entero más próximo, pues los tamaños poblacionales solo pueden ser números enteros positivos.

Los modelos actuales del elemento teórico de Ricker se definen como sigue: $\mathbf{M}(\mathbf{R}): x$ es una población de tipo Ricker $(x \in \mathbf{M}(\mathbf{R}))$ syss existen $N, T, \lambda_{0}, k$ y $b$ tales que

(1) $x=\left\langle N, T, \lambda_{0}, k, b\right\rangle$

(2) $x \in \mathbf{M}_{\mathbf{p}}(\mathbf{R})$

(3) $n\left(t_{2}\right)=\operatorname{Trunc}\left(\lambda_{0} n\left(t_{1}\right) e^{\left(-b n\left(t_{1}\right)\right)}\right)$

siendo $n\left(t_{1}\right)$ y $n\left(t_{2}\right)$ los tamaños de las poblaciones en los intstantes $t_{1}$ y $t_{2}$ respectivamente.

Argumentar que la red logística y la que representa el tiempo como variable discreta pueden capturarse bajo la forma de una única red, identificando un elemento teórico en un nivel más elevado, como el indicado, es poco plausible. Incluso aunque este argumento pudiera ser formalmente correcto, es una estrategia artificial porque el nuevo elemento teórico no es un elemento empírico de las prácticas ecológicas. Como se mostrará más adelante, el uso de constelaciones de elementos no pertenecientes a la misma red teórica se extiende no solo a elementos de redes afines, como en el caso presente, sino de redes con marcos conceptuales de representación más diferenciados. Por ello, aun cuando pudiera aceptarse la estrategia de integrar la reconstrucción del tiempo como variable discreta y como variable continua en una única red, encontraríamos dificultades para interpretar con ella la complejidad de determinadas prácticas científicas, como son las ecológicas, objeto de este artículo. Supondría reducir impropiamente esa complejidad a algunos patrones de prácticas científicas bien fijadas por la imagen de ciencia normal kuhniana. 
Como puede verse, el marco conceptual (definido por la clase $\mathbf{M}_{\mathbf{p}}$ ) de este elemento teórico es notablemente diferente al de los elementos teóricos que conforman la red logística, aunque, como aquellos, incluye un elemento, $k$, que impone un límite teórico máximo al tamaño que puede alcanzar la población, de ahí que los ecólogos lo consideren "análogo" al elemento logístico de Verhulst.

Lo mismo puede decirse del elemento teórico asociado a la ley de BevertonHolt. Esta ley tiene la siguiente forma:

$$
N_{t+1}=\frac{\lambda_{0} N_{t}}{1+b N t}
$$

El elemento teórico de Beverton-Holt puede reconstruirse así:

$\mathbf{M}_{\mathbf{p}}(\mathbf{B H}): x$ es una población de tipo Beverton-Holt potencial $\left(x \in \mathbf{M}_{\mathbf{p}}(\mathbf{B H})\right)$ syss existen $N, T, \lambda_{0}, k$ y $b$ tales que

(1) $x=\left\langle N, T, \lambda_{0}, k, b\right\rangle$

(2) $N$ es un conjunto finito, no vacío y $N \subseteq \mathbb{N}$

(3) $T$ contiene exactamente dos elementos $\left(T=\left\{t_{1}, t_{2}\right\}\right)$

(4) $\lambda_{0} \in \mathbb{R}$

(5) $k \in \mathbb{N}$ y $k<|N|$

(6) $b=\lambda_{0}-1 / k$

(7) Trunc: $\mathbb{R} \rightarrow \mathbb{N}$

Y para la clase de los modelos actuales:

$\mathbf{M}(\mathbf{B H}): x$ es una población de tipo Beverton-Holt $(x \in \mathbf{M}(\mathbf{B H}))$ syss existen $N, T, \lambda_{0}$, $k$ y $b$ tales que

(1) $x=\left\langle N, T, \lambda_{0}, k, b\right\rangle$

(2) $x \in \mathbf{M}_{\mathbf{p}}(\mathbf{B H})$

(3) $n\left(t_{2}\right)=\operatorname{Trunc}\left(\lambda_{0} n\left(t_{1}\right) / 1+b n\left(t_{1}\right)\right)$

Igualmente, existe un elemento teórico asociado al denominado mapa logístico o mapa cuadrático, cuya ley tiene la forma:

$$
N_{t+1}=\lambda_{0} N_{t}\left(1-\frac{N_{t}}{k}\right)
$$

y, por tanto, puede reconstruirse modeloteóricamente como sigue:

$\mathbf{M}_{\mathbf{p}}(\mathbf{C})$ : $x$ es una población de tipo cuadrático potencial $\left(x \in \mathbf{M}_{\mathbf{p}}(\mathbf{C})\right)$ syss existen $N, T$, $\lambda_{0}$ y $k$ tales que

(1) $x=\langle N, T, \lambda, k\rangle$

(2) $N$ es un conjunto finito, no vacío y $N \subseteq \mathbb{N}$

(3) $T$ contiene exactamente dos elementos $\left(T=\left\{t_{1}, t_{2}\right\}\right)$

(4) $\lambda_{0} \in \mathbb{R}$

(5) $k \in \mathbb{N}_{\text {y }} k<|N|$

(6) Trunc: $\mathbb{R} \rightarrow \mathbb{N}$

La clase de sus modelos actuales es reconstruible así: 
$\mathbf{M}(\mathbf{C}): x$ es una población de tipo cuadrático $(x \in \mathbf{M}(\mathbf{C}))$ syss existen $N, T, \lambda_{0}$ y $k$ tales que

(1) $x=\left\langle N, T, \lambda_{0}, k\right\rangle$

(2) $x \in \mathbf{M}_{\mathbf{p}}(\mathbf{C})$

(3) $n\left(t_{2}\right)=\operatorname{Trunc}\left(\lambda_{0} n\left(t_{1}\right)\left(1-n\left(t_{1}\right) / k\right)\right)$

Este último elemento teórico es conocido por haber introducido el caos en la ecología (May 1974a, 1974b). En efecto, el mapa logístico muestra un comportamiento que depende básicamente del valor del parámetro $\lambda_{0}$, y va desde el crecimiento sigmoideo, cuando los valores de $\lambda_{0}$ son bajos, al caos, cuando $\lambda_{0}>$ 2,449 , pasando por los ciclos amortiguados y los ciclos con límites estables cuando los valores de $\lambda_{0}$ son intermedios (Figura 7).

Figura 7
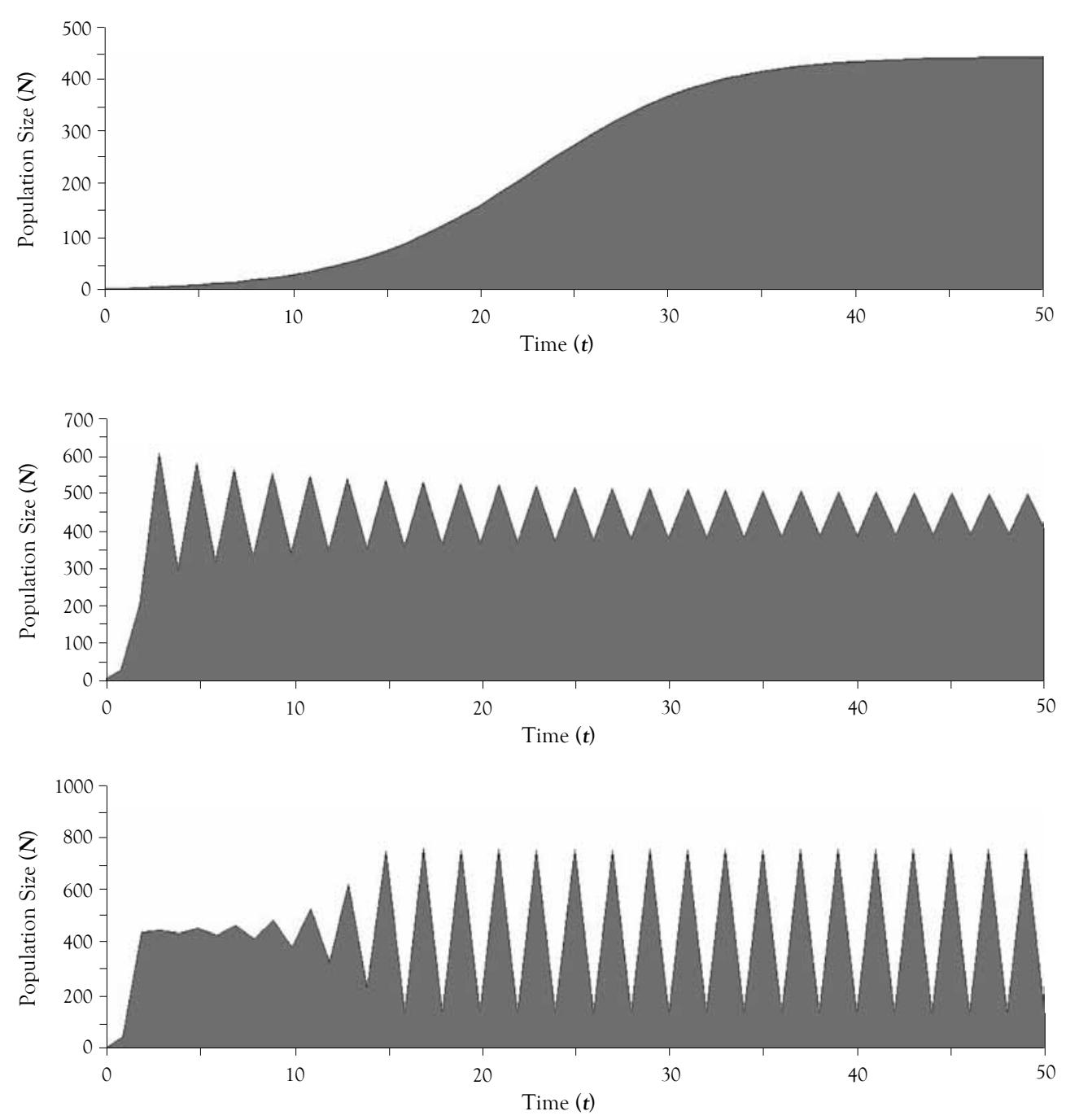


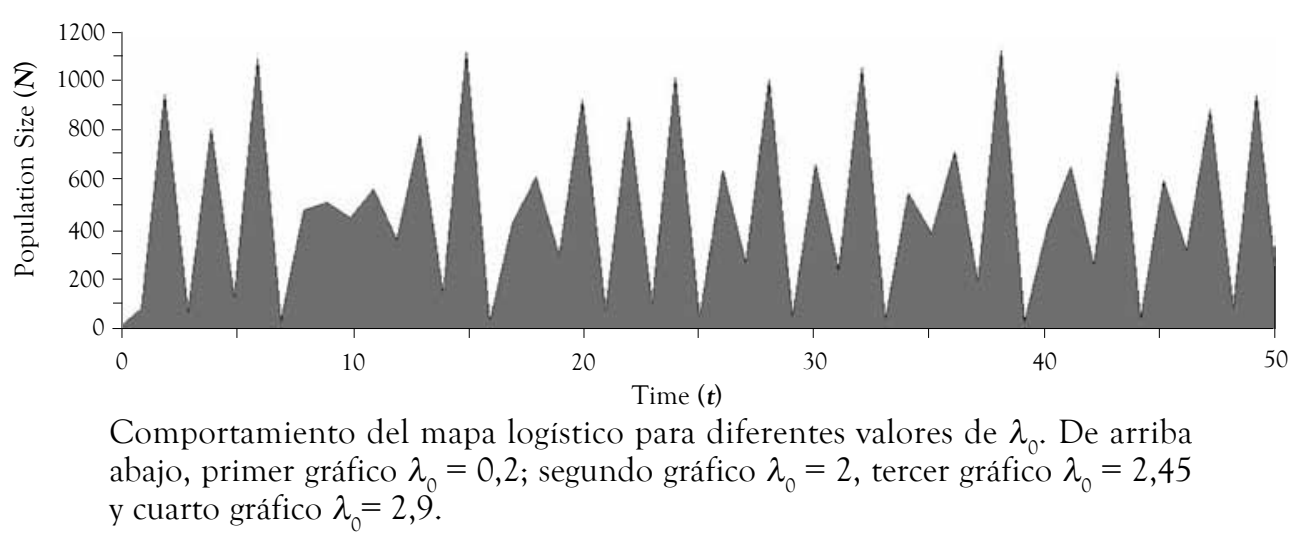

El elemento teórico de Ricker representa situaciones de comportamiento semejantes al del mapa logístico, mientras que el elemento de Beverton-Holt muestra un comportamiento muy similar al del elemento logístico de Verhulst, pues el único resultado a largo plazo es la aproximación monótona a un punto de equilibrio estable. Esto se atribuye al hecho de que la forma en que los elementos teóricos de Ricker y del mapa logístico introducen la dependencia de la densidad es más extrema que en el elemento de Beverton-Holt (Hastings 2005). Así pues, y esta es la información relevante obtenida de la constelación de los elementos de dos redes teóricas distintas, estos tres elementos teóricos, aunque basados, al igual que el elemento logístico de Verhulst, en la idea de que toda población posee un tamaño máximo que no le es posible superar (al menos no por mucho tiempo), muestran un comportamiento diferente de sus respectivos análogos en tiempo continuo, más diferente cuanto más extrema es la forma en que incorporan la dependencia de la densidad. Esto apoya la idea de que los retrasos temporales tienen un efecto desestabilizador en relación a los cambios en la densidad poblacional. Dicho de otro modo, el aquí presentado es un ejemplo de que es la comparación o la puesta en común de los resultados de una constelación teórica, esto es, de elementos de redes teóricas diferentes, lo que lleva a resultados robustos. Los ecólogos teóricos confrontan sistemáticamente elementos pertenecientes a diferentes redes teóricas, para ver si sus predicciones teóricas coinciden y, en los casos en que así es, consideran que la coincidencia de resultados apoya la idea de que las predicciones de los elementos teóricos comparados son robustas. Así pues, la idea fundamental sobre la que se basa la noción de resultado robusto es la de que, si los resultados de varios elementos de una constelación teórica que representan un mismo proceso pero poseen diferente forma o estructura convergen, es menos arriesgado concluir que la influencia del proceso estudiado es, precisamente, la que representan los elementos teóricos de la constelación. Es decir, solo comparando entre sí elementos teóricos diferentes (pertenecientes a redes teóricas diferentes), puede saberse si los resultados a los que apuntan uno o varios elementos teóricos son robustos, de ahí que la comparación entre elementos teóricos de una constelación sea una práctica habitual en la ecología de poblaciones. 
Una constelación teórica es un conjunto de al menos dos elementos teóricos, algunos de los cuales pertenecen a teorías o redes teóricas distintas (de ahí que sus modelos potenciales sean diferentes), pero comparten (al menos) parte del dominio de aplicaciones intencionales. Es más, dado que el dominio de aplicaciones intencionales se define como subconjunto del conjunto de modelos potenciales parciales $\left(\mathbf{I} \subseteq \mathbf{M}_{\mathbf{p p}}\right.$ ) (Balzer, Moulines \& Sneed 1987, pp. 86-89), el hecho de que la intersección entre dominios de aplicaciones intencionales sea un conjunto no vacío implica que también los modelos potenciales parciales de los elementos teóricos que componen la constelación teórica compartirán (al menos) algunos elementos comunes, es decir, $\mathbf{M}_{\mathbf{p p}}\left(\mathbf{T}_{\mathrm{i}}\right) \cap \mathbf{M}_{\mathbf{p p}}\left(\mathbf{T}_{\mathrm{j}}\right) \neq \varnothing$ (por ejemplo, todos los elementos teóricos que se han analizado en esta sección poseen un conjunto de tamaños poblacionales entre los componentes de sus modelos potenciales y modelos potenciales parciales).

Laconstelaciónteórica puededefinirse modeloteóricamente, entonces, comosigue: Sea $\boldsymbol{C}=\left\langle\mathbf{T}_{1}, \ldots, \mathbf{T}_{\mathbf{n}}\right\rangle$ un conjunto de elementos teóricos $\mathbf{T}_{1}=\left\langle\mathbf{M}_{\mathrm{p}}\left(\mathbf{T}_{1}\right), \mathbf{M}_{\mathrm{pp}}\left(\mathbf{T}_{1}\right)\right.$, $\left.\mathbf{M}\left(\mathbf{T}_{1}\right), \mathbf{G C}\left(\mathbf{T}_{1}\right), \mathbf{G} \mathbf{L}\left(\mathbf{T}_{1}\right), \mathbf{I}\left(\mathbf{T}_{1}\right)\right\rangle, \ldots, \mathbf{T}_{\mathrm{n}}=\left\langle\mathbf{M}_{\mathbf{p}}\left(\mathbf{T}_{\mathrm{n}}\right), \mathbf{M}_{\mathbf{p p}}\left(\mathbf{T}_{\mathbf{n}}\right), \mathbf{M}^{\mathrm{p}}\left(\mathbf{T}_{\mathrm{n}}\right), \mathbf{G C}\left(\mathbf{T}_{\mathrm{n}}\right)\right.$, $\left.\mathbf{G L}\left(\mathbf{T}_{\mathrm{n}}\right), \mathbf{I}\left(\mathbf{T}_{\mathrm{n}}\right)\right\rangle$, identificados en los términos estructuralistas habituales. $\boldsymbol{C}$ es una constelación teórica syss $\exists \boldsymbol{A}$ tal que:

(1) $\boldsymbol{A}=\left\langle\mathbf{T}_{\mathrm{i}}, \mathbf{T}_{\mathrm{j}}\right\rangle$ y $\boldsymbol{A} \subset \boldsymbol{C}$

(2) $\mathbf{M}_{\mathbf{p}}\left(\mathbf{T}_{\mathrm{i}}\right) \neq \mathbf{M}_{\mathbf{p}}\left(\mathbf{T}_{\mathrm{j}}\right)$

(3) $\mathbf{I}_{\mathrm{i}} \cap \mathbf{I}_{\mathrm{j}} \neq \varnothing$

Obviamente, esta es una versión idealizada de constelación teórica. Una definición más realista de las constelaciones debería considerar constricciones sobre otros componentes estructurales de los elementos teóricos, como por ejemplo GL: para dar cuenta, por ejemplo, de las relaciones existentes entre la tasa de crecimiento per capita, $r$, en los elementos teóricos de la red logística y el parámetro $\lambda_{0}$, que también es una tasa de crecimiento independiente de la densidad, en los elementos de la red logística en tiempo discreto.

En todo caso, la situación que se ha analizado en esta sección no representa sino una instanciación muy sencilla de confrontación de resultados ofrecidos por elementos de una constelación teórica con el fin de llegar a conclusiones más robustas que las que pueden obtenerse de la combinación de elementos de una única red. En la sección siguiente se considerará otro caso, más complejo, de la actividad ecológica a partir de constelaciones teóricas que dan origen a resultados robustos.

\section{Constelaciones complejas: elementos teóricos sensibles a las diferencias individuales y la práctica de simulaciones informáticas}

El desarrollo de la modelización actual en la ecología de poblaciones está estrechamente vinculado a la construcción de "modelos" complejos construidos para ser implementados en un ordenador. Característicamente, estos "modelos infor- 
máticos" no han desplazado a los componentes de las redes presentadas anteriormente, elementos teóricos mucho más sencillos pero que siguen siendo considerados útiles (Grimm 1999, Railsback 2001). ¿Por qué?

El uso de la informática ha permitido, entre otras cosas, introducir en las teorías ecológicas la variabilidad individual. Hasta hace relativamente poco, la mayor parte de las teorías ecológicas ignoraba las diferencias entre individuos o, a lo sumo, distinguía entre unas pocas clases de edades o de tamaños. En cambio, durante las dos últimas décadas, es cada vez más habitual el uso de lo que los ecólogos denominan "modelos basados en el comportamiento de los individuos" o IBMs (individual based models). Son estos elementos teóricos en los que a cada uno de los individuos que forman la población se le atribuye una serie de características particulares, con lo que la población deja de ser un agregado de individuos idénticos o un agregado de individuos de unos pocos tipos.

En esta sección procuraremos, en primer lugar, mostrar que los elementos teóricos a los que da lugar este tipo de investigación se insertan en redes teóricas distintas a las dos que se han analizado en las secciones anteriores y que, en segundo lugar, frente a la imagen de la ciencia normal de Kuhn y el estructuralismo, los ecólogos incorporan también estos nuevos elementos en el análisis de las constelaciones que construyen para obtener resultados robustos.

Entre los IBMs destinados a estudiar los factores que regulan las dinámicas de las poblaciones individuales se encuentra el desarrollado por Uchmański para estudiar cómo el reparto de recursos y el tipo de competencia afectan la regulación de las poblaciones (Uchmański 1999). Uchmański comienza construyendo y analizando un primer elemento teórico "nulo" en el que todos los individuos son iguales. A partir de él, modifica este primer elemento introduciendo diferencias individuales de diverso tipo. Así, hace que la mortalidad sea aleatoria y que, pese a existir recursos suficientes, solo algunos de los individuos lleguen a reproducirse. $\mathrm{O}$ introduce diferencias en el crecimiento individual debidas a la competencia por los recursos. Comparando el comportamiento de esos tres elementos teóricos, Uchmański concluye, en claro contraste con lo que sugieren otras teorías como las que previamente aquí se han analizado, que, para que el crecimiento de la población se encuentre regulado, es necesario que exista algún tipo de variabilidad individual.

Nos detendremos a continuación en la propuesta de Uchmański, en tanto que red teórica distinta de las precedentes. La red teórica desarrollada por Uchmański tiene como características fundamentales de su marco conceptual $\left(\mathbf{M}_{\mathbf{p}}\right)$ el tratar de manera individualizada cada uno de los componentes de la población y el representar de manera explícita los recursos utilizados por cada uno de ellos. Para ello, establece, en primer lugar, que los individuos crecen de acuerdo con la siguiente ecuación:

$$
\frac{d w}{d t}=a_{1} w^{b_{1}}-a_{2} w^{b_{2}}
$$

donde $w$ es el peso del individuo, $b_{1}$ describe el aumento de masa corporal debido a la asimilación de recursos y $b_{2}$ describe las pérdidas debidas a la respiración. 
La asimilación de recursos y la respiración son iguales cuando el peso del individuo es igual al peso final dado por la ecuación siguiente:

$$
w_{\text {end }}=\left(\frac{a_{1}}{a_{2}}\right)^{\frac{1}{b_{2}-b_{1}}}
$$

El coeficiente $a_{1}$ varía dependiendo de las condiciones de los recursos, $V$; la relación entre $a_{1}$ y $V$ viene dada por la ecuación de Michaelis-Menten:

$$
a_{1}=a_{1 \max } \frac{V}{V+\delta}
$$

donde $\delta$ es la constante de semisaturación y $a_{1 \max }$ es el valor máximo del parámetro $a_{1}$. Los parámetros $a_{2}$ y $b_{2}$ son constantes, lo que implica que la tasa de respiración del individuo es constante e independiente de la cantidad de recursos de la que el individuo dispone.

La cantidad de descendientes que produce un individuo, $z$, es proporcional a la diferencia entre el peso final y un umbral de peso $w_{\text {thr }}$ :

$$
z=\operatorname{Trunc}\left(c\left(w_{\text {end }}-w_{\text {thr }}\right)\right)
$$

donde $c$ es una constante y la función Trunc redondea un número real hasta su entero más próximo. Por tanto, se considera que un individuo muere sin descendencia si su peso es menor que $w_{\text {thr }}$.

Por último, para analizar las dinámicas de la población formada por individuos con esas características, en la que todos los individuos son iguales, la disponibilidad de recursos no varía durante cada generación y las generaciones no se solapan se utiliza la siguiente ecuación:

$$
N_{t+1}=\sum_{i=1}^{N_{t}} z_{i}
$$

y los recursos disponibles en la siguiente generación se calculan de la siguiente manera:

$$
V_{t+1}=V_{t}+g-N_{t u a 1 w_{\text {end }}^{b_{1}}}^{b_{1}}
$$

donde $g$ es el flujo de recursos al sistema, $N_{t}$ la cantidad de individuos en la generación $t$, el parámetro $u$ es inversamente proporcional al coeficiente de eficiencia en la asimilación y el término $a_{1} w_{\text {end }}^{b_{1}}$ - es una aproximación del consumo acumulado durante la vida del individuo.

Reconstruiremos modeloteóricamente el elemento teórico más complejo propuesto por Uchmański: ofreceremos un esquema de su reconstrucción, suficiente a nuestro juicio para mostrar la naturaleza claramente diferente respecto de los elementos teóricos considerados hasta ahora:

$\mathbf{M}_{\mathbf{p}}(\mathbf{U C H})$ : $x$ es una población de Uchmański potencial $\left(x \in \mathbf{M}_{\mathbf{p}}(\mathbf{U C H})\right)$ syss existen

$I, T, V, Z, b_{1}, b_{2}, \delta, a_{1}, a_{1 \max }, a_{2}, c, g, u, w, w_{\text {thr }} \mathrm{y} w_{\text {end }}$ tales que

(1) $x=\left\langle I, T, V, Z, b_{1}, b_{2}, \delta, a_{1}, a_{1 \max }, a_{2}, c, g, u, w, w_{\text {thri }}, w_{\text {end }}\right\rangle$

(2) $I$ es un conjunto finito, no vacío y $I=\left\{i_{1}, \ldots, i_{n}\right\}$ 
(3) $T$ es un conjunto y $T$ contiene dos elementos $T=\{t, t+1\}$

(4) $N$ es un conjunto finito, no vacío y $\mathrm{N} \subset \mathbb{N}$

(5) $Z$ es un conjunto finito, no vacío y $Z \subset \mathbb{N}$

(6) $V$ es un conjunto finito, no vacío y $V \subset \mathbb{R}$

(7) $b_{1}: I \rightarrow \mathbb{R}$ para todo $i \in I: b_{1}(i)>0$

(8) $b_{2}: I \rightarrow \mathbb{R}$ para todo $i \in I: b_{2}(i)>0$

(9) $\delta \in \mathbb{R}^{+}$

(10) $a_{1}: I \rightarrow \mathbb{R}$ para todo $i \in I: 0<a_{1}(i) \leq a_{1 \max }$

(11) $a_{1 \text { max }}: I \rightarrow \mathbb{R}$ para todo $i \in I: 0<a_{1}(i) \leq a_{1 \text { max }}$

(12) $a_{2}: I \rightarrow \mathbb{R}$ para todo $i \in I: a_{2}>0$

(13) $c \in \mathbb{R}$

(14) $g \in \mathbb{R}$

(15) $u=1 / b_{1}$

(16) $w: I \times T \rightarrow \mathbb{R}$ para todo $i \in I: w(i)>0$ y $w$ es continua y derivable

(17) $w_{\text {thr }} \in \mathbb{R}$

Como puede observarse, en lo que a sus modelos potenciales respecta, este elemento teórico sería muy diferente de cualquiera de los demás elementos teóricos que aquí se han analizado, no solo por su mayor complejidad, sino también porque incorpora elementos que ninguno de los modelos potenciales de los anteriores elementos teóricos incluirían, como, por ejemplo, un conjunto I formado por individuos cada uno de los cuales llevaría asociada una serie de variables y parámetros como su peso, la capacidad de asimilar nutrientes del medio y, por tanto, aumentar de peso, un umbral de peso mínimo que necesita superar para poder reproducirse, etc. Es más, la teoría de Uchmański tiene la particularidad de que representa de manera explícita la cantidad de recursos de que dispone cada individuo de la población, algo que ninguno de los elementos teóricos anteriores hace. Como consecuencia, en esta teoría el tratamiento de la natalidad es radicalmente diferente con respecto a las teorías poblacionales clásicas. Esta deja de ser representada únicamente mediante un parámetro y es tratada como una función dependiente del estado de cada individuo que, a su vez, depende de la cantidad de recursos disponible en el medio y de la capacidad individual para asimilarlos. Lo mismo ocurre con la mortalidad y con el tratamiento de la competencia.

La red teórica de Uchmański contiene los elementos teóricos que representan las diferentes leyes que Uchmański requiere satisfacer a cada uno de los factores identificados en $\mathbf{M}_{\mathbf{p}}(\mathbf{U} \mathbf{C H})$. Las posibilidades son, pues, varias. Una de ellas sería la siguiente:

$\mathbf{M}(\mathbf{U C H}): x$ es una población de Uchmański $(x \in \mathbf{M}(\mathbf{U C H}))$ syss existen $I, T, V, Z$, $b_{1}, b_{2}, \delta, a_{1}, a_{1 \max }, a_{2}, c, g, u, w, w_{\text {thr }} \mathrm{y} w_{\text {end }}$ tales que

(1) $x=\left\langle\mathrm{I}, T, V, Z, b_{1}, b_{2}, \delta, a_{1}, a_{1 \max }, a_{2}, c, g, u, w, w_{\mathrm{thr}}, w_{\text {end }}\right\rangle$

(2) $x \in \mathbf{M}_{\mathbf{p}}(\mathbf{U C H})$

(3) $D w(i)=a_{1}(i) w(i)^{b_{1}(i)}-a_{2}(i) w(i)^{b_{2}(i)}$ 
(4) $w_{\text {end' end }}=\left(\frac{a_{1}(i)}{a_{2}(i)}\right)_{V}^{\frac{1}{b_{2}(i)-b_{1}(i)}}$

(5) $a_{1}(i)=a_{1 \max }(i) \frac{V}{V+\delta}$

(6) $z(i)=\operatorname{Trunc}\left(c\left(w_{\text {end }}(i)-w_{\text {thr }}\right)\right)$

(7) $n_{t+1}=\sum_{i=1}^{n_{t}} z_{i}$

(8) $v_{t+1}=v_{t}^{i=1}+g-n_{t} u(i) a_{1}(i) w_{\text {end }}(i)^{b_{1}}$

Ahora bien, como se acaba de apuntar, la red teórica que Uchmański propone puede irse ampliando en la medida en que la investigación sugiera la necesidad de introducir nuevos elementos teóricos, asociados a nuevas leyes, para investigar nuevas cuestiones. Esto implicaría que las cláusulas 3 a 8 podrían variar y que podrían, incluso, añadirse nuevas cláusulas.

Así, cuando Uchmański modifica su elemento teórico inicial para hacer que la mortalidad afecte de manera diferencial a los individuos que conforman la población, lo hace asignando al nuevo elemento teórico asociado la siguiente función:

$$
m=m_{\max } \frac{\alpha}{\alpha+V}
$$

donde $m_{\max }$ es la mortalidad máxima, $V$ la cantidad de recursos disponible y $\alpha$ una constante. Igualmente, la competencia se incorpora al elemento teórico correspondiente haciendo que los individuos tengan diferentes pesos corporales y la cantidad de recursos que pueden asimilar dependa de esta variable, de acuerdo con esta otra función:

$$
a_{1}^{i}=a_{1}^{i}\left(w_{0}^{i}, V\right)
$$

que se puede especificar de diferentes maneras para representar diferentes tipos de competencia en diferentes elementos teóricos, dando así lugar a una red teórica más compleja cuantas más formas de competencia se quieran incorporar a la teoría.

Así pues, es claro que los elementos teóricos de Uchmański integran una red diferente de la red logística o la red formada por los elementos teóricos que representan el crecimiento poblacional mediante ecuaciones en diferencias. Esta nueva red puede ampliarse con otros elementos teóricos que representan de manera explícita el comportamiento de los individuos que conforman la población, como en (Uchmański 2000) donde se considera la influencia de diferentes formas de reparto de recursos en las dinámicas de las poblaciones.

Finalmente, la red de Uchmański muestra que la regulación de las poblaciones exige la existencia de diferencias entre los individuos que componen las poblaciones. Muestra, en concreto, que la estabilidad de las poblaciones es mayor cuanto mayor es la diferencia entre individuos, pues las poblaciones formadas por individuos idénticos no parecen estar reguladas, las poblaciones en las que 
existe una variabilidad limitada entre individuos muestran indicios de regulación débil y aquellas en las que la variabilidad individual es alta (existen diferencias entre todos los individuos de la población) la regulación de las poblaciones es efectiva y estas persisten durante largo tiempo.

Sin embargo, el aspecto relevante a destacar aquí radica en que es la constelación teórica integradora de elementos de la red de Uchmański y de otras redes la que permite considerar como un resultado robusto la afirmación de que el factor que más influye en la regulación y la persistencia de las poblaciones es la existencia o no de algún tipo de variabilidad individual. Entre esas otras redes se sitúan, sin duda, los diversos elementos teóricos de Eomnicki, que hacen depender de las diferencias individuales en la obtención de recursos la estabilidad y la persistencia de las poblaciones (Łomnicki 1988, 2009). Un elemento teórico de Eomnicki representa los efectos del reparto desigual de recursos entre individuos a partir de cuatro elementos diferentes, cada uno de los cuales representa de manera diferente el reparto de recursos en poblaciones cuyas generaciones no se solapan (si bien se considera que todos los individuos que componen la población son genéticamente iguales y de la misma edad y sexo). En este caso, la estabilidad y la persistencia de una población exigen un reparto desigual de los recursos. Otro elemento teórico de la red de Łomnicki asume que las generaciones se solapan y en este caso puede concluirse que la relación entre la distribución desigual de los recursos entre diferentes individuos de la población y la estabilidad y la persistencia de las poblaciones es general. Justamente, un elemento teórico de la red de Uchmański representa en tiempo continuo la situación de este segundo elemento de Łomnicki, haciendo dependiente del parámetro emigración la estabilidad o no de las dinámicas de las poblaciones: con emigración o sin ella, las dinámicas poblacionales presentan puntos de equilibrio estables (lo que demuestra que en la estabilidad de las poblaciones no influye únicamente de la emigración, sino también de algún otro factor, probablemente la existencia de diferencias individuales en la obtención de alimentos) (Uchmański 1983). Junto a estos elementos más complejos, también se integran en esta constelación teórica elementos más sencillos como el que vincula la distribución desigual de los individuos de la población a la competencia por el alimento y al reparto desigual de este entre los individuos de la población (Kimmel 1986).

En suma, en la literatura ecológica son cada vez más abundantes este tipo de elementos teóricos relativamente complejos, que constituyen redes que toman en consideración aspectos hasta ahora descuidados por las teorías poblacionales clásicas. Ahora bien, lo que nos interesa resaltar aquí es que, en las prácticas "normales" de los ecólogos, estos nuevos elementos teóricos no reemplazan a elementos -o a redes- teóricos anteriores, demasiado sencillos para representar adecuadamente los procesos que tienen lugar en las poblaciones y, por tanto, parcialmente obsoletos. Por el contrario, los ecólogos combinan simultáneamente en constelaciones teóricas elementos de diversas redes (complejas o más sencillas).

Los elementos son complementarios en el siguiente sentido. Los elementos teóricos complejos tienden a ser opacos, más opacos cuanto más complejos. Quie- 
re decirse con esto que en los elementos complejos en los que se incluye una cantidad relativamente grande de variables y parámetros, suele ser difícil aprehender los mecanismos por los cuales el parámetro (o parámetros) del elemento se comporta(n) de una manera determinada (Grimm \& Railsback 2005, pp. 363 369). En cambio, en los elementos teóricos más sencillos esto es relativamente fácil. Así pues, la construcción e integración de elementos teóricos de características muy diferentes (pertenecientes a redes distintas) y de muy diferentes niveles de complejidad en asociaciones modelísticas como las constelaciones, que se aplican a un dominio común de aplicación, tiene por objetivo comprender de manera cada vez más profunda cómo diferentes factores y procesos influyen en el comportamiento de los sistemas estudiados. Los elementos integrados en las constelaciones teóricas procuran aproximaciones simbióticas en la solución a problemas comunes.

\section{Conclusión}

En esta contribución se han analizado algunos aspectos de las prácticas de construcción teórica en la ecología de poblaciones con un triple fin. En primer lugar se ha buscado hacer evidente, bien que de manera bastante intuitiva, la estructura de los denominados "modelos" de la ecología de poblaciones a partir de su reconstrucción como elementos teóricos integrados en redes y relacionados entre sí por relaciones de especialización. En segundo lugar, se ha querido poner de manifiesto, mediante un ejemplo sencillo, la importancia del análisis e integración en constelaciones teóricas de elementos de redes diferentes que, sin embargo, incluyen la influencia de un mismo factor o proceso a la hora de asegurar que las consecuencias extraídas sobre la influencia de los mencionados factores o procesos sean robustas. Por último, se ha querido mostrar que, en la ecología de poblaciones, es cada vez más usual la construcción de elementos teóricos relativamente complejos, pertenecientes a redes muy diferentes a las preexistentes, cuya utilidad depende, en gran medida, de la existencia de elementos teóricos más sencillos, pertenecientes a otras redes, con los que pueden ser comparados. En suma, se ha querido hacer razonable la idea de que en la ecología de poblaciones es una estrategia habitual y una práctica epistémicamente fecunda la comparación de los resultados de elementos teóricos de redes diferentes, con el fin de llegar a conclusiones robustas sobre la influencia de determinados factores en las dinámicas de las poblaciones naturales.

Habitualmente, esta práctica exige agrupar en una misma unidad epistémica elementos pertenecientes a diferentes redes teóricas. Esos agrupamientos característicos de la ecología de poblaciones se integran en lo que hemos denominado constelaciones teóricas, unidades epistémicas que aplican diversos elementos teóricos de redes distintas a un dominio común de aplicación. No es posible adquirir resultados robustos analizando los factores que influyen en las dinámicas de las poblaciones con base en el marco conceptual provisto por una única red teórica, dada la compleja naturaleza de las poblaciones. De ahí que se requiera la in- 
tegración de elementos de diversas redes en una imagen de la ciencia normal distinta de la ofrecida por Kuhn. En la imagen de las prácticas científicas que aquí se propone, el ecólogo no dispone, en términos estructuralistas, de una teoría, de una red teórica, sino de constelaciones teóricas.

\section{Bibliografía}

Balzer, W., Moulines, C.U. y J.D. Sneed (1987), An Architectonic for Science. The Structuralist Program, Dordrecht: Reidel.

Berryman, A.A. (1999), Principles of Population Dynamics and their Applications, Cheltenham: Stanley Thornes.

Diederich, W., Ibarra, A. y T. Mormann (1989), "Bibliography of Structuralism”, Erkenntnis 30(3): 387-407.

Diederich, W., Ibarra, A. y T. Mormann (1994), "Bibliography of Structuralism II (1989-1994 and Additions)", Erkenntnis 41(3): 403-418.

Gotelli, N. J. (2001), A Primer for Ecology, 3a ed., Sunderland: Sinauer.

Grimm, V. (1999), "Ten Years of Individual-Based Modelling in Ecology: What We Have Learned and What Could We Learn in the Future?", Ecological Modelling 115(2-3): 129. 148.

Grimm, V. y S.F. Railsback (2005), Individual-Based Modeling and Ecology, Princeton: Princeton University Press.

Hall, C.A.S. (1988), "An Assessment of Several of the Historically Most Influential Theoretical Models Used in Ecology and of the Data Provided in their Support", Ecological Modelling 43(1-2): 5-31.

Hastings, A. (2005), "Unstructured Models in Ecology: Past, Present, and Future”, en Cuddington, K. y B. Beisner (eds.), Ecological Paradigms Lost: Routes of Theory Change, Amsterdam: Elsevier, pp. 9-29.

Jensen, A.L. (1975), “Comparison of Logistic Equations for Population Growth”, Biometrics 31(4): 853-862.

Kimmel, M. (1986), “Does Competition for Food Imply Skegness?”, Mathematical Biosciences $80(2): 239-264$.

Kingsland, S.E. (1995), Modeling Nature: Episodes in the History of Population Ecology, 2a ed., Chicago: The University of Chicago Press.

Lande, R., S. Engel y B. Sather (2003), Stochastic Population Dynamics in Ecology and Conservation, Oxford: Oxford University Press.

Levins, R. (1966), "The Strategy of Model Building in Population Biology", American Scientist 84(4): 421-431.

Lewontin, R.C. (2003), "Building a Science of Population Biology", en Singh, R.S. y M.K. Uyenoyama (eds.), The Evolution of Population Biology, Cambridge: Cambridge University Press, pp. 7-20.

Eomnicki, A. (1988), Population Ecology of Individuals, Princeton: Princeton University Press. 
Łomnicki, A. (2009), "Scramble and Contest Competition, Unequal Resource Allocation, and Resource Monopolization as Determinants of Population Dynamics”, Evolutionary Ecology Research 11: 371-380.

May, R.M. (1974a), Stability and Complexity in Model Ecosystems, 2a ed., Princeton: Princeton University Press.

May, R.M. (1974b), "Biological Populations with Nonoverlapping Generations: Stable Points, Stable Cycles, and Chaos", Science 186: 645-647.

Moulines, C.U. (1996), “Structuralism: The Basic Ideas”, en Balzer, W. y C.U. Moulines (eds.), Structuralism Theory of Science: Focal Issues, New Results, Berlin: de Gruyter, pp. 1-13.

Moulines, C.U. (2010), "Metatheorical Structuralism: a General Program for Analyzing Science", Axiomathes 20(2-3): 255-268.

Odenbaugh, J. (2003), "Complex Systems, Trade-Offs, and Theoretical Population Biology: Richard Levins' "Strategy of Model Building in Population Biology” Revisited”, Philosophy of Science 70(5): 1496-1507.

Railsback, S.F. (2001), "Concepts from Complex Adaptative Systems as a Framework for Individual-Based Modelling”, Ecological Modelling 139: 77-62.

Turchin, P. (2003), Complex Population Dynamics: A Theoretical/Empirical Synthesis, Princeton: Princeton University Press.

Uchmański, J. (1983), "The Effect of Emigration on Population Stability: A Generalization of the Model of Regulation of Animal Numbers, Based on Individual Differences", Oikos 41(1): 49-56.

Uchmański, J. (1999), "What Promotes Persistence of a Single Population: An IndividualBased Model”, Ecological Modelling 115: 227-242.

Uchmański, J. (2000), "Resource Partitioning among Competing Individual and Population Persistence: An Individual-Based Model”, Ecological Modelling 131: 21-32.

Vandermeer, J.H. y D.E. Goldberg (2003), Population Ecology: First Principles, Princeton: Princeton University Press.

Wombat, W.C. (1980), "Randomness and Perceived-Randomness in Evolutionary Biology", Synthese 43(2): 287-329.

Weisberg, M. (2006), “Robustness Analysis”, Philosophy of Science 73(5): 730-742. 